
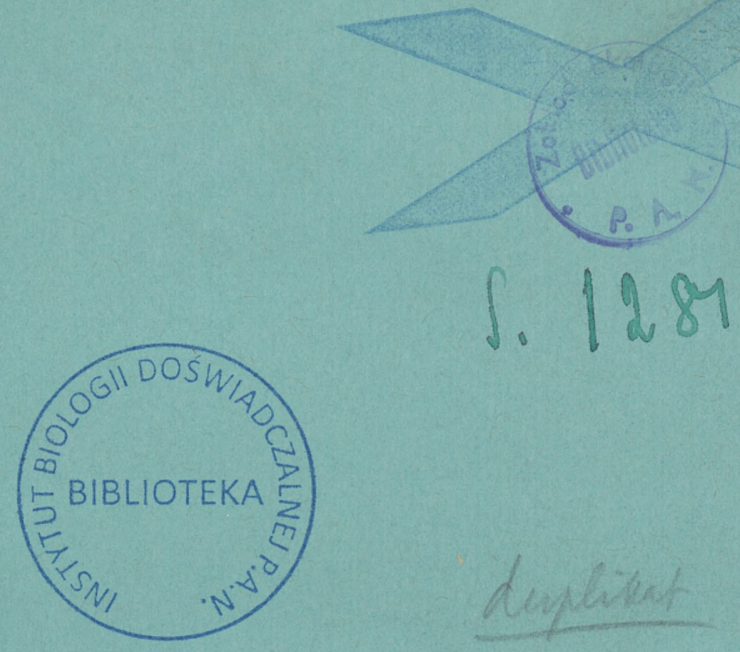

duplivat

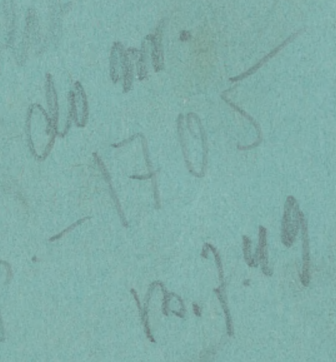

rcin.org.pl 

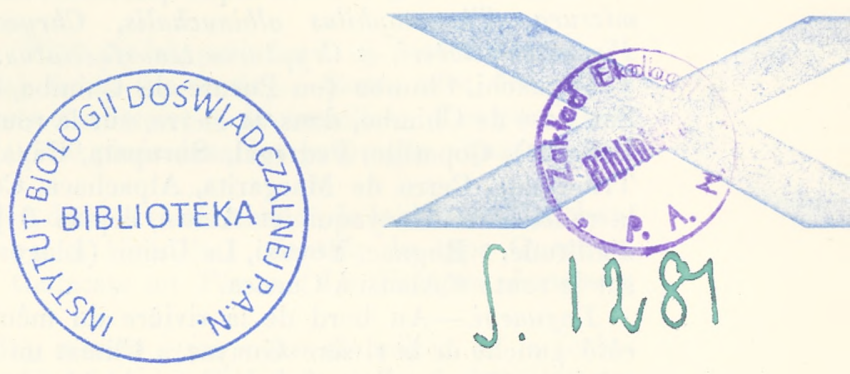

[From the Proceedings of the Zoological Society of London, May 6, 1884.]

Deuxième liste des Oiseaux recueillis dans l'Ecuadeur occidental par MM. Stolzmann et Siemiradski. Par le Comte Hans de Berlepsch, C.M.Z.S., et L. TaczaNowski, C.M.Z.S.

\section{(Plate XXIV.)}

Les oiseaux de cette collection ont été recueillis dans les localités suivantes.

Guayaquil._Climat chaud et sec; la contrée parsemée en partie de mimoses et de cactus, couverte en partie de forêts et de broussailles, qui perdent en entier leur verdure en été. Au sud de la ville s'étendent les manglars, couvrant les ilots de la rivière Guayas et l'ile de 
Puna. Les oiseaux caractéristiques pour cette localité sont:--Myrmia micrura, Thamnophilus albinuchalis, Chrysomitris siemiradzkii, Picumnus sclateri, et Crypturus transfasciatus.

Yaguachi, Chimbo (ou Puente de Chimbo, pour le distinguer du San Jose de Chimbo, dans la sierra, sur la route entre Guaranda et Podegas), Copatillo, Pedregal, Surupata, Cayandeled, Chaguarpata, Tribulpata, Cerro de Margarita, Alpachaca, Cechce sont situés sur Ia route entre Guayaquil et Alausi, depuis 0 jusqu'à 10,000 pieds d'altitude. Bugnac, Yoyaxi, La Union (Llagos) et Sical se trouvent sur la route d'Alausi à Cuenca.

Yaguachi.-Au bord de la rivière du même nom, tributaire du côté gauche de la rivière Guayas. Climat un peu plus humide que celui de Guayaquil; végétation semblable, mais la faure un peu différente, on n'y voit plus d'espèces d'oiseaux cités plus haut, mais en revanche on y trouve le Campylorhynchus balteatus, Picumnus olivaceus, et Thamnophilus transandeanus.

Puente de Chimbo, d'où venait la plus grande partie des oiseaux de la liste précédente, est la dernière station du chemin de fer allant de Yaguachi ( 800 pieds) le long de rivière Chimbo. Forêt vierge, humide; la température s'y baisse la nuit jusqu'au $17^{\circ}$ Centigrade. Oiseaux caractéristiques sont :-Cephalopterus penduliger, Heliothrix barroti, Thalurania hypochlora, Picumnus olivaceus, Odontophorus erythrops, et Penelope ortoni. Grande diversité de la faune dans les différentes saisons à cause des migrations des espèces de la côte pendant le desséchement des forêts, p. e. Heliomaster albicrissa.

Copatillo (1000 pieds), Pedregal (2600 pieds).-Forêts chaudes, faunes mélangées comme à Chimbo et à Cayandeled.

Cayandeled (4500 pieds).--Forêt humide, température modérée, s'abaissant la nuit jusqu'au $12^{\circ} \mathrm{C}$. Végétation différente ce celle de Chimbo, les lianes y manquent presque complètement; en revanche commencent les fourrés de buissons dans la forêt; les arbres moins élevés, les fougères arborescentes remplacent les palmiers; les nectandres et les ficus prédominent. Oiseaux caractéristiques:Cynanthus cyanurus, Chamcepetes goudoti, nombreaux Callistes, la limite supérieure du domaine du Myobius stellatus, Cephalopterus penduliger, et Ramphocelus icteronotus, l'inférieure du Scytalopus magellanicus, Chloronerpes fumigatus et des Buarremons vrais. Les excursions y ont été entreprises sur une grande surface vericale, en bas jusqu'à Surupata (3700 pieds), El Placer (2600 pieds) et Pedıegal (2600 pieds) toutes les trois fermes; plus haut jusqu'à Chaguarpata (5800 pieds), Tribulpata (7000 pieds), localités inhabitées, forêts serranes, où on trouve les premières Lafresnayes, les Metallures et la Psittospiza riefferi.

Alpachaca (10,000 pieds), dernières parties forestières et commencement de la puna; la température s'y baisse la nuit jusqu'au $6^{\circ} \mathrm{C}$, souvent il y neige. Profusion des oiseaux mouches (Diphlogcena, Lesbia, Petasophora et autres), voitigeant dans les broussailles à fleurs continuelles.

Alausi ( 7500 pieds). - Chef-lieu du canton de ce nom, situé au bord d'une vallée non boisée au pied d'Azuagu, au bord de la rivière $[2]$ 
de ce nom, tributaire de la rivière Chancan, du système du Rio de Chimbo.

Laguna de Ticksan (9000 pieds), petit Jac à $1 \frac{1}{2}$ lieue d'Alausi.

Cechce (9600 pieds), ferme (hacienda).- Sierra avec des parties forestières, des bouquets d'arbres et de broussailles. Les excursions s'étendaient jusqu'à 10,700 pieds dans les paturages alpestres couverts de la Stipa ichu.

Bugnac (6000 pieds), dans la vallée chaude de la rivière Chanchan, sur la route entre Alausi et Chunche. Contrée du type Quechua, semblable à celle de Callacate au Pérou. La Tanagra darwini y abonde.

Yoyaxi (9000 pieds), marais assez vaste au milieu de la forêt; climat froid comme à Alpachaca et Cechce, mais les forêts élevées y vont plus haut que dans les localités citées, où à 8000 pieds commencent les broussailles naines (des myrtes et des lauriers) au milieu desquels il y a des groupes d'arbres plus élevés.

La Union ou Llagos (9000 pieds).-Ferme située au voisinage de la ville Chunche (Province Asnay-Cuenca). Forêts vierges, élevées, froides, domaine du Tapir et de l'Ours. Oiseaux, absents à Cechce, Cyanocorax turcosa et Penelope montagnii.

Toutes ces localités sont situées sur le versant occidental des Cordillères.

\section{TURDIDE.}

*1. Catharus fuscater (Lafr.).

Quatre nâles et une femelle recueillis à Cayandeled en janvier et février. Iris blanc.

\section{Catharus dryas (Gould).}

Cinq mâles recueillis à Pedregal (2600 pieds), à Placer (2800 pieds) et à Cayandeled en janvier et février. Iris brun foncé.

Tous ces oiseaux ainsi que ceux de Chimbo ont le jaune du dessous beaucoup plus vif que les oiseaux de Huambo (Pérou septentrional) recueillis en mars et en avril, et n'ont pas la gorge maculée de noir comme ces derniers; la couleur du dos est distinctement olivâtre, ce qui ne se voit pas dans les oiseaux péruviens. Tous ces exemplaires sont d'une taille un peu moins forte (l'aile pliée est de 86--90 mill., tandis que dans les oiseaux péruviens elle est de 97).

3. Turdus ignobilis maculirostris, Berl. et Tacz.

Deux mâles adultes d'El Placer (2800 pieds), recueillis le 27 et le 29 février.

Ces oiseaux présentent tous les caractères de la coloration semblables à ceux de Chimbo.

*4. Turdus serranus, Tsch.

Un mâle et trois femelles de Cayandeled et de Chaguarpata, recueillis entre le 20 janvier et le 18 février. Iris brun foncé.

L'unique mâle de cette collection est d'une taille moins forte que l'oiseau du Pérou central, et s'en distingue par le noir moins intense 
et moins lustré en dessus, parfaitement mat en dessous. Il a la première remige distinctement plus longue, dépassant plus l'extrémité des grandes tectrices; la queue moins étagée; le bec plus long et plus élargi à la base, d'un rouge orangé. Longueur de l'aile 121 , queue 111 , bec 30 , tarse 35 , différence entre les rectrices externes et les médianes 11 mill., tandis que chez l'oiseau péruvien la longueur de l'aile est de 132 , queue 117 , bec 29 , tarse 37 , différence entre les rectrices externes et les médianes $17 \mathrm{~mm}$.

\section{Troglodytide.}

5. Henicorhina hilaris, Stolz. MS.

H. prostheleucæ similis, sed differt pectore griseo lavato (nec niveo), abdominis lateribus totis latissime brunneo-rufis, abdomine medio albido rufescente perfuso; plumis alulæ spuria rufescente vix marginatis; alis obsolete transfasciatis.

ô et ㅇ ad. Sommet de la tête brun, bordé des deux côtés d'une bande noire; dos roux brunâtre foncé, passant sur le croupion en roux plus vif et plus clair que celui de la $H$. prostheleuca; côtés de la tête noirs, bordés en dessus d'une longue bande sourcilière blanche commençant aux narines et descendant jusqu'au bas des côtés du cou, la partie renfermée entre l'œil et les narines colorée légèrement d'ocreux : les joues et la région auriculaire maculés également comme dans les espèces voisines de taches longitudinales blanches en laissant une large bande d'un noir pur derrière l'œil, séparant le sourcil de cette maculature; gorge blanche lavée légèrement d'ocreux; toute la poitrine blanchâtre enduite de cendré grisâtre plus fortement sur les côtés qu'au milieu; les côtés de tout l'abdomen largement roux-brunâtre foncé; milieu gris blanchâtre lavé de roussâtre, passant plus ou moins au roux sur la région anale; souscaudales d'un roux clair. Ailes d'un brun roussâtre extérieurement, à barbes externes des remiges transversées de bandes noirâtres beaucoup moins nettes, moins prononcées et moins larges que chez la H. prostheleuca, sans rien de blanchâtre sur les primaries; les plumes de l'aile batarde bordées finement de roussâtre au lieu de blanc; barbe interne des remiges schistacée; sousalaires grises tachetées de blanchâtre. Queue brune rayée en travers de noir moins régulièrement que dans l'espèce citée. Bec aussi long mais moins fort que celui de la $H$. prostheleuca, noirâtre; pattes brunes. Iris brun foncé.

$$
\begin{aligned}
& \text { ๙. Longueur de l'aile 55, queue 29, bec } 20 \text {, tarse } 25 \mathrm{~mm} \text {. } \\
& \text { ㅇ. } \quad \text {, } 50 \text { " } 20 \text {, } 19 \text { " } 24 \text {, }
\end{aligned}
$$

Cette forme diffère de l'oiseau de Chimbo, que nous avons nommé dans la liste précédente $H$. leucophrys, par la couleur de la poitrine non cendrée, le roux beaucoup plus repandu sur le dessous du corps et surtout beaucoup plus prolongé sur les côtés du haut de l'abdomen; il a le bec également long. Les oiseaux du Pérou septentrional (Huambo) sont identiques à ceux de Chimbo, mais l'oiseau du Pérou central diffère de tous les autres par le bec beaucoup plus court et 
beaucoup moins élargi à la base, ainsi que par le manque complet de raies noires sur les remiges.

Huit mâles et une femelle de Cayandeled, deux paires de Chaguarpata (5700 pieds) et un mâle de Pedregal (2800 pieds), recueillis en janvier, février et mars.

*6. Thryothorus euophrys, Scl.

Mâle adulte et une jeune femelle en mue de Cechce recueillis en mai, un jeune mâle de Cayandeled tué le 16 janvier. Iris brun.

Ce jeune mâle diffère des adultes par le sommet de la tête d'un gris de souris; par le manque complet de taches noires sur la poitrine et le milieu de l'abdomen; par le fond de l'abdomen plus roussâtre, la tache postoculaire brune au lieu de noir; le bec moins long, pâle, à mandibule inférieure blanchâtre; une taille moins forte.

7. Thryothorus mystacalis, Scl.

Un mâle pris par Stolzmann à Cayandeled, le 27 février.

8. Troglodytes furvus (Gm.).

Deux mâles recueillis par Stolzmann à Surupata en février et mars.

Identiques aux oiseaux péruviens (T. audax, Tsch.), et également distincts de ceux de Cayenne par le manque complet de raies foncées au dos, et par une taille moins forte. Ils nous paraissent être différents de l'oiseau de Guayaquil.

\section{Troglodytes solstitialis, Scl.}

Une paire recueillie par Stolzmann à Cayandeled le 16 janvier. Iris brun.

*10. Cistothorus brunneiceps, Salv. Ibis, 1881, p. 129, pl. iii. f 1 .

Une paire d'adultes et trois jeunes recueillis par Stolzmann à Cechce (10,000 pieds) en mai. Iris brun grisâtre foncé.

Distincts des oiseaux péruviens par le sommet de la tête d'un brun unicolore sans stries fauves, les plumes du croupion traversées de quelques raies noirâtres bien distinctes, la gorge lavée de fauve et non blanc pur comme chez les oiseaux cités, le blanc du milieu du ventre également coloré légèrement de fauve, tandis que chez l'oiseau cité il est pur et bien distinct du roux des flancs. La dimension est un peu moins forte dans l'oiseau de l'Ecuadeur occidental, l'aile est longue de $45 \mathrm{~mm}$., tandis que les péruviens l'ont de 47 . Le jeune en premier plumage ressemble en tout au jeune du Cistothorus péruvien, et présente la même longueur de l'aile. En général toutes ces formes des Cistothorus sont aussi alliées entre elles qu'elles ne doivent être distinguées que conme des races locales.

\section{Motacillide.}

*11. Anthus bogotensis, Scl.

Deux mâles et une femelle de Cechce, pris par Stolzmann en avril et mai,

Proc. Zood. Soc.-1884, No. XX. 


\section{Mniotiltide.}

12. Parula pitiayumi pacifica, Berl.

Un mâle de Surupata ( 3700 pieds) et une femelle de Cayandeled, recueillis en mars et en janvier. Iris brun foncé.

Ces oiseaux de l'envoi récent, ainsi que ceux de Chimbo, ont beaucoup moins de blanc sur les tectrices supérieures des ailes et les rectrices externes (le mâle de Suruputa n'a presque point de cette couleur sur les ailes) que tous les exemplaires de l'orient que nous avons examiné. Les oiseaux de l'Ecuadeur occidental présentent donc sous ce rapport une transition entre la vraie $P$. pitiayumi et la $P$. inornata, Baird, de l'Amérique centrale, ou plutôt la race de cette dernière de Veragua qui n'a qu'une seule bande blanche sur l'aile, tandis que la $P$. inornata de Guatemala n'en a point. L'oiseau de Tumbez (Pérou nord-occidental) a les deux raies transalaires bien marquées.

*13. Dendreca blackburnieg (Gm.).

Deux oiseaux adultes de Cayandeled, pris le 16 février et le 17 mars. Iris brun foncé.

Les premiers exemplaires furent observés dans cette localité le 15 février (Siemiradzki).

*14. Basileuterus coronatus (Tsch.).

Cinq oiseaux des deux sexes de Cayandeled recueillis en janvier et mars, un mâle de Tribulpata (6500 pieds) pris en mars, et une fernelle de Cechee d'avril.

Ces oiseaux sont d'une taille un peu moins forte que ceux du Pérou septentrional (aile plus courte de 4-5 mm.), les oiseaux du Pérou central sont encore un peu plus forts. Dans la coloration ils se distinguent des péruviens par la nuance du dos tirant au roussâtre, plus encore sur les ailes et la queue, en dessous la nuance rousse se manifeste aussi plus ou moins sur les sonscaudales et les côtés du bas ventre; le jaune de l'abdomen est aussi un peu plus fort.

15. Basileuterus fraseri, Scl. P. Z. S. 1883, p. 653, pl. lxi.

Un mâle recueilli par Stolzmann à Pedregal le 27 février.

*16. Basileuterus tristriatus, Tsch.

Trois mâles et deux femelles recueillis par Stolzmann en février à Cayandeled, Pedregal (3000 pieds) et à Placer (2800 pieds).

Ces oiseaux diffèrent des péruviens par le jaune du dessous moins beau, la bande sourcilière d'une couleur plus sale, la gorge enduite d'ocreux pâle, une taille moins forte.

*17. Basileuterus nigricristatus (Lafr.).

Deux mâles recueillis par Stolzmann à Cayandeled et à Cechce en janvier et avril.

*18. Setophaga bairdi, Salv.

Une paire de Cechce recueillie par Stolzmann le 6 mai. [6] 
19. Setophaga verticalis (d'Orb. et Lafr.).

Deux mâles et une femelle recueillis par Stolzmann à Cayandeled en janvier et février.

*20. Geothlypis semiflaya, Scl.

Une femelle prise par Stolzmann à Placer (2800 pieds) le 30 janvier.

\section{VIREONIDE.}

*21. Vireosylvia josephe, Scl. P. Z. S. 1859 p. 173 (décrite de Pallatanga).

Quatre mâles recueillis à Pedregal et à Cayandeled en janvier, février et mars, et un jeune sans étiquette. Iris brun très foncé.

\section{Hirundinide.}

\section{*22. Atticora murina, Cass.}

Un oiseau adulte sans indication de sexe et un jeune en mue, de Ticsan (7000-9000 pieds), recueillis en mai et en juin.

La description de Hirundo cinerea, Gm., n'est pas assez complète pour qu'elle puisse être appliquée à cette espèce. Elle se distingue de la péruvienne par la couleur du dessous distinctement plus foncée et le manque presque complet d'éclat vert sur les petites tectrices alaires.

\section{Cerrebide.}

*23. Diglossa albilateralis, Lafr.

Dix mâles et quatre femelles de Cayandeled, de Cechce et de Chaguarpata (5200 pieds), recueillis entre le 10 janvier et le 10 mai.

Ces oiseaux sont un peu plus petits (surtout à queue plus courte) que les oiseaux de Bogota dans la collection Berlepsch, mais sans aucune différence dans la coloration. La même remarque peut être appliquée aux oiseaux du Pérou, qui sont aussi d'une taille plus forte; la coloration du mâle est la même; mais les femelles de l'Ecuadeur présentent une petite différence dans la couleur du dessous du corps, où le milieu du ventre est coloré distinctement de jaunâtre, le dessus du corps est plus olivâtre. Ces oiseaux sont cependant en plumage tout frais, tandis que les péruviens sont en plumage usé.

\section{*24. Diglossa aterrima, Lafr.}

Trois mâles de Cechce, recueillis en avril et en mai.

*25. Diglossa personata, Fras.

Deux mâles de Chaguarpata pris en février et mars; deux mâles, une femelle et un mâle très jeune de Cayandeled pris en janvier et février, un mâle de Tribulpata ( 8000 pieds) du 8 mars, et une femelle de Cechce prise en avril. Iris rouge, rouge cerise et carminé, gris chez le jeune. 
Identiques aux oiseaux de Bogota dans la collection Berlepsch.

*26. Conirostrum fraseri, Scl. P. Z. S. 1858, p. 752 (décrit de Cuenca).

Trois mâles et deux femelles de Cechce recueillis en avril et en mai.

\section{*27. Conirostrum atrocyaneum, Lafr.}

Un mâle de Cayandeled et cinq femelles de Chaguarpata et de Pedregal, recueillis depuis janvier jusqu 'en avril.

Ces oiseaux sont identiques à ceux de Pérou. Le type de Lafresnaye venait de Rio Napo. L'oiseau de Pallatanga de la collection Fraser, nommé dans la liste (P.Z.S. 1859, p. 138) C. albifrons, serait probablement le $C$. atrocyaneum.

\section{TANAGRIDE。}

\section{*28. Euphonia Nigricollis (Vieill.)}

Deux jeunes mâles recueillis par Stolz!nann à Cayandeled dans la moitié de mars.

29. Euphonia xanthogastra, Sundev.

Trois mâles adultes, deux femelles et deux jeunes mâles recueillis à Cayandeled et à Pedregal en janvier et février.

*30. Euphonia saturata, Cab.

Un mâle adulte et un jeune prenant sa livrée d'adulte recueillis par Stolzmann à Surupata (3600-3700 pieds) en janvier et février.

Ces oiseaux ont le jaune du sommet de la tête un peu moins intense et le jaune roussâtre du ventre moins pur que dans l'oiseau de Turnbez; la couleur du dos et des ailes plus violette et moins bleue.

31. Calliste aurulenta, Lafr.

Quatre mâles, trois jeunes et un oiseau sans indication de sexe recueillis à Cayandeled en janvier, février et mars.

Ces oiseaux de l'Ecuadeur occidental ont les bordures des plumes dorsales, des tectrices alaires et des remiges secondaires d'un jaune d'or plus intense et plus rougeâtre que les oiseaux de Bogota, qui ont ces bordures plus jaunes ou même verdâtres; en revanche ils ont la couleur de la tête et du dessous moins intense et moins rougeâtre. Les dimensions sont les mêmes.

*32. Calliste Ruficervix, Prev.

Trois paires d'oiseaux adultes de Cayandeled (4000 pieds) recueillis en janvier, février et mars. Iris brun foncé.

Ces oiseaux s'accordent en général avec un exemplaire de Bogota de la collection Berlepsch; ils ont seulement les couleurs un peu plus vives, le bleu de ciel du corps plus pur et moins verdâtre; le bord supérieur des tectrices auriculaires d'un or roussâtre comme celui de l'occiput et non blanchâtre comme dans l'oiseau cité; la bande bleuâtre frontale moins large. Les ailes et la queue sont plus courtes, mais le bec paraît être plus long.

$[x]$ 
*33. Calliste nigroviridis (Lafr.).

Trois mâles, deux femelles et un jeune recueillis à Cayandeled depuis le 31 janvier jusqu'au 28 février, et une femelle de Chaguarpata (5700 pieds) du 23 février. Iris brun foncé.

Semblables aux oiseaux de Bogota, mais moins bleuâtres sur la tête, le croupion et la poitrine; un peu plus petits. La race péruvienne est considérablement plus forte.

*34. Calliste lunigera, Scl.

Trois mâles, trois femelles et deux oiseaux sans indication de sexe de Cayandeled, recueillis depuis le 16 janvier jusqu'au 6 mars. Iris brun foncé ou de terre de Sienne.

*35. Calliste venusta, Scl.

Une femelle prise par Stolzmann à Cayandeled le 10 fevrier.

*36. Pecilothraupis lunulata (DuBus.).

Trois mâles et deux femelles de Cechce recueillis entre le 28 avril et le 6 mai. Iris brun foncé.

Intermédiaires entre la $P$. lunulata de Bogota et la $P$. atricrissa de Quito. Un mâle du 2 mai a les souscaudales terminées distinctement de bordures rouges, tandis que les autres individus n'en ont rien de rouge ou des légères indications de cette couleur. Les oiseaux de Quito ont toujours les sous-caudales noires en entier. Ceux de Bogota ont les plumes bordées plus largement de rouge que les oiseaux de Cechce. En outre il n'y a pas d'autre différence entre les oiseaux de ces trois localités.

*37. Compsocoma sumptuosa cyanoptera (Cab.).

Un mâle et trois femelles de Cayandeled et de Chaguarpata, recueillis en janvier, février et mars. Iris brun foncé ou brun rougeâtre.

L'intensité du bleu aux bords des remiges varie un peu. Une femelle du 16 février l'a plus clair que les autres, d'un bleu de ciel. Il paraît que les oiseaux de Quito ont ce bleu un peu plus intense que la majorité des oiseaux de Cayandeled.

*38, Pipridea melanonota venezuelensis (Scl.).

Un mâle pris par Stolzmann à Cayandeled le 16 mars. Iris rouge brunâtre.

Identique aux oiseaux péruviens.

*39. Dubusia taniata (Boiss.).

Une paire de Cechce, recueilis par Stolzmann en avril et mai. Iris rouge cerise. Nos oiseaux sont d'une taille moins forte que l'exemplaire de la Colombie (Musée de Varsovie), ils ont l'aile longue de 89 mill., tandis que ce dernier l'a de 98 , et se distinguent par le bleu du dos non violâtre; les taches bleues du sourcil et du front n'ont rien de violet et ne le prennent que très légèrement et dans une seule direction de la lumière. Tous les oiseaux du Musée Berlepsch pro- 
venant de l'Ecuadeur et de la Nouvelle Grenade ont la taille rapprochée à nos oiseaux de Cechce, et aucun ne présente pas de bleu aussi foncé que celui du Musée de Varsovie.

*40. Tanagra cyanocephala, Lafr. et d'Orb.

Cinq mâles et trois femelles de Cayandeled et Surupata depuis le 14 janvier jusqu'au 28 février. Iris brun foncé.

Ces oiseaux ont les tectrices souscaudales d'un vert, olive, dans quelques-uns à bordures jaune verdâtres, plus ou moins prononcés. Chez la $T$. auricrissa de Bogota les souscaudales sont d'un jaune presque pur, le jaune des sousalaires est aussi plus intense, et le vert olive du dos plus brunâtre et plus jaunâtre que dans nos oiseaux. Ils sont parfaitement identiques aux oiseaux du Pérou central et septentrional, et présentent les mêmes variétés sous le rapport de la présence et du développement des bordures jaunes sur les souscaudales. L'oiseau de Medellin se distingue le plus, et surtout par la nuance des parties inférieures du corps beaucoup plus foncée que chez tous les autres.

41. Tanagra cana, Sws.

Un mâle et deux femelles de Cayandeled, recueillis par Siemiradzki le 13 et 17 mars.

*42. Tanagra darwini, Bp.

Un mâle adulte et une femelle très jeune recueillis à Bugnac par Siemiradzki en juin.

43. Ramphocklus icteronotus, Bp.

Deux mâles et une femelle recueillis par Siemiradzki à Cayandeled et à Pinampunga en janvier et mars.

*44. Nemosia ornata, Scl.

Trois paires de Cayandeled, de Pedregal (2830 pieds) et de Bugnac (6400 pieds) recueillis en janvier, février, mars et mai. Iris brun foncé.

*45. Chlorospingus nigrifrons (Lawr.), Ibis, 1875, p. 384.

Un mâle de Cechce (10,000 pieds) pris par Stolzmann en avril.

Cet oiseau présente une très petite différence des oiseaux péruviens dans la coloration de la tête, dont le front est d'une couleur plus foncée, les côtés de la tête d'une coleur beaucoup plus foncée et presque uniforme, et la bande sourcilière plus blanche. Outre cela on ne voit aucune différence.

*46. Chlorospingus Phaocephalus, Scl. et Salv. P. Z. S. 1877, p. 521 (décrit de Jina et de Chillanes).

Treize mâles adultes et une femelle de Surupata et de Cayandeled recueillis en janvier, février et mars, et un jeune de Chimbo pris en septembre. Iris rouge de sang, palissant aussitôt après la mort de l'oiseau en couleur carnée ou rouge ocreux chez les autres; le jeune l'a brun grisâtre. 
Le jeune ne se distingue des adultes que par le sommet de la tête, et ses côtés lavés distinctement d'olive, qui dans certaines directions de la lumière prend un ton presque semblable à celui du dos; le gris sale de la gorge sans aucune trace d'ocreux; et la bande pectorale d'un olive sale, semblable à celui des flancs.

*47. Chlorospingus ochraceus, sp. n. (Plate XXIV. fig. I.)

Ch. capite supra cum nucha fusco-cinereo, vitta superciliari vix pallidiore, lateribus capitis brunneis; dorso ex olivaceo pallide brunneo, uropygio rufescentiore; corpore subtus ochraceo badio, hypochondriis brunnescentibus, subcaudalibus intense rufis; tectricibus alorum minoribus cinereis; majoribus et mediis dorso concoloribus; remigibus extus et cauda rufescentibus; subalaribus ochraceis. Rostrum nigricanti-corneum; pedes brunnei; iris brunnea.

ㅇ ad. Sommet de la tête d'un cendré foncé tirant très peu sur l'olivâtre, bordé des deux côtés d'une raie sourcilière d'une nuance un peu moins foncée à peine distincte, dont la partie antéoculaire est légèrement colorée d'ocreux; le dos est d'un brun peu foncé tirant un peu sur l'olive, et passant au roussâtre sur la partie postérieure du croupion; côtés de la tête bruns; tout le dessous du corps d'un ocreux rougeâtre, plus pur et plus clair au bas de la gorge et sur le milieu de l'abdomen, enduit largement de brunâtre sur les flancs et la poitrine; souscaudales d'un roux plus intense que le ventre. Petites tectrices alaires d'un cendré un peu bleuâtre, les autres de la couleur du dos; remiges brunes, les primaries et les secondaires bordées largement à l'extérieur de brun olivâtre clair, tirant sur le roussâtre sur la moitié basale des pennes; les tertiaires et la barbe externe des secondaires voisines d'une couleur semblable à celle du dos; sousalaires ocreuses; bord interne des remiges fauve. Queue brun roussâtre tirant un peu sur l'olive. Bec corné noirâtre; pattes brunes ; iris brun. Longueur de l'aile 77, queue 70, bec 19, tarse $20 \mathrm{~mm}$.

Une autre femelle, probablement moins adulte, avec le sommet de la tête et les petites tectrices alaires non cendrés mais d'un olive un peu plus verdâtre que le dos, a la strie sourcilière plus distincte et tirant au jaune blanchâtre.

Forme voisine du Ch. melanotis, Scl, de Bogota, mais avec la région auriculaire brune au lieu de noire, dos brun olivâtre au lieu d'ardoisé ; queue et ailes brun roussâtres à l'extérieur au lieu d'olives ; milieu du ventre sans blanc et les souscaudales plus rousses.

Deux femelles tuées par Siemiradzki à Cayandeled et à Chaguarpata, le 16 janvier et le 5 mars.

*48. Buarremon assimilis, Boiss.

Deux mâles de Cechce, du 5 et 12 mai, identiques aux oiseaux de Bogota et du Pérou septentrional.

*49. Buarremon inornatus, Scl. et Salv. Ibis 1879, p. 426.

Un mâle adulte recueilli par Siemiradzki à Cayandeled, le 17 janvier. Iris terre de sienne brulée. 
Le Musée de Varsovie possède un oiseau acquis du feu Verreaux, nommé par Jules Verreaux B. brunneinuchus, et étiquetté comme provenant du Mexique, semblable en tout à notre oiseau de l'Ecuadeur, sans aucune trace de collier noir, mais qui s'en distingue par le brun du sommet de la tête plus sombre et sans bordure latérale, plus claire et plus vive, semblables à celle du $B$. brunneinuchus; l'olive du dos plus sombre sans aucune trace de vert jaunâtre sur la nuque. Les dimensions sont les mêmes.

*50. Carenochrous spodionotus (Scl. et Salv.), Ibis 1879, p. 425 (décrit de Guapulo, Calacali et Sical).

Deux mâles et une femelle de Cechce tués le 4 mai. Iris brun rougeâtre foncé.

Un de ces oiseaux, du Musée Berlepsch, a une petite tache jaune au commencement et au dessus des freins, qui sont noirs. Cette particularité se trouve quelquefois dans cette espèce, tandis que le $C$. comptus (Scl. et Salv.) a une tache frontale plus grosse et plus distincte. Le dernier a aussi la calotte rousse plus pâle et plus atténuée en arrière. (Voyez l'Ibis, l. c.)

\section{Carenochrous leucopterus (Jard.).}

Cinq mâles et six femelles de Cayandeled, de Cechce (10,000 pieds), de Surupata (3800 pieds) et un jeune en premier plumage de Bugnac (6400 pieds) recueillis en février, mars et mai. Iris brun rougeâtre.

Presque tous ont une tache noirâtre au front entre le blanc des freins, qui manque complètement chez un mâle de Cechce.

Le jeune en premier plumage se distingue des adultes par la couleur du dos tirant au brunâtre, le roux du sommet de la tête sale, le noir des côtés de la tête beaucoup moins foncé, la tache postnasale moins nette, des grosses stries noirâtres sur la poitrine, les flancs d'un gris roussâtre au lieu de cendré, sous-caudales moins rousses, mandibule inférieure d'un jaune orangée.

*52. Psittospiza riefferi (Boiss.).

Une paire de Chaguarpata et de La Union ( 8700 pieds) de férrier et de juin. Iris roux (couleur des parties environnantes).

Identiques aux oiseaux de Bogota.

\section{Fringillidda.}

\section{Pheucticus chrysogaster (Less.).}

Deux mâles de Cechce (10,000 pieds) et de Bugnac (6000 pieds), et une femelle de Cayandeled (7500 pieds), pris en février, avril et juin. Iris brun foncé. Ces oiseaux s'accordent en général avec les oiseaux de Quito (Musée Berlepsch); ils sont seulement un peu plus petits, surtout le bec est moins gros. Nous avons déjà indiqué la différence de la coloration entre les oiseaux de Quito et de Chimbo dans notre article précédent. Le mâle de Bugnac est coloré comme ceux de Quito; il paraît que la femelle de Cayandeled diffère de celle de Chimbo en ce qu'elle a les taches noires du dos et des côtés de la $[12]$ 
tête beaucoup plus larges et plus prononcées; le dos supérieur est presque tout à fait noir. Si ces différences seraient constantes on pourrait séparer la forme des localités plus élevées comme une race locale.

\section{Spermophila gutturalis olivacea, Stolzm.}

Sept mâles et une femelle de Cayandeled, recueillis depuis janvier jusqu'en février.

\section{*55. Spermophila luctuosa, Lafr. et d'Orb.} mai.

Deux femelles recueillis par Stolzmann à Bugnac (6000 pieds) en

56. Spermophila Pauper, Stolzm. MS. (Plate XXIV. fig. 2.)

S. obscura, Tacz. P.Z.S. 1880, p. 199. Berl. et Tacz. P.Z.S., 1883 , p. 550 .

Trois mâles adultes de Cayandeled recueillis par Stolzmann, identiques aux oiseaux de Chimbo et du Pérou septentrional(Callacate) et non à la $S p$. obscura du Pérou central. Cette forme se distingue de cette dernière par une taille moins forte, le bec plus robuste et plus large, les couleurs moins foncées et particulièrement en dessous, le blanc beaucoup plus repandu au milieu du ventre, la nuance roussâtre plus ou moins distincte sur les ailes. Les dimensions sont les suivantes:-

t. Long. totale 121-125, vol 182, aile 51-53, queue 35-43, bec 9 , tarse $15 \mathrm{~mm}$.

오. Long. totale 118 , vol 171 , aile $50-53$, queue $37-38$, bec 9 , tarse $14 \mathrm{~mm}$.

\section{*57. Catamenia analoides, Lafr.}

Un mâle et deux femelles recueillis par Stolzmann à Cechce, Bugnac et sur la route d'Alausi à Bugnac (6500 pieds), en mai.

*58. Catamenia homochroa, Sel. P.Z.S. 1858, p. 552 (décrit de Matos).

Sept mâles et trois femelles de Cechce recueillis en avril et mai. Les oiseaux du Pérou septentrional nommés par Taczanowski $C$. inornat $\alpha$ sont identiques à ceux de Cechce; ils ont les mêmes dimensions et les mêmes couleurs. La vraie $C$. inornata (Lafr.), dont un exemplaire Bolivien se trouve au Musée Berlepsch, est une forme très voisine, mais beaucoup plus grande. On ne voit aucune différence de coloration entre ces deux formes.

Les mâles de Cechce, aile $66 \frac{1}{2}$, queue 58-60, bec $9-10$, tarse $20 \frac{1}{2} \mathrm{~mm}$.

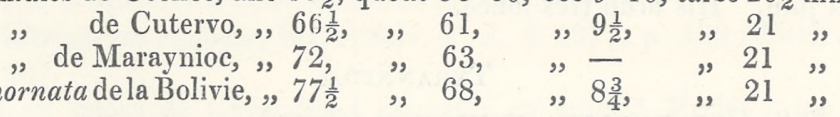

*59. Catamblyrhynchus diadema (Lafr.).

Trois mâles et deux femelles de Cayandeled et de Chaguarpata, recueillis en janvier, février et mars. 
Identiques aux oiseaux péruviens; la seule différence que présente l'unique mâle péruvien du Musée de Varsovie consiste dans la présence d'une bande rousse complète en travers du croupion, ce qui ne se voit sur les oiseaux de la Nouvelle Grénade et chez nos oiseaux de l'Ecuadeur occidental; dans tous ces oiseaux la couleur rousse n'est visible que sur les côtés du croupion.

60. Volatinia Jacarina splendens (Vieill.).

Un mâle recueilli par Stolzmann à Placer (2800 pieds) le 30 janvier.

*61. Phrygilus alaudinus (Kittl.).

Un mâle pris par Stolzmann à Cechce le 4 mai. Iris brun foncé.

*62. Phrygirus plebeius, Tsch.

Une femelle prise par Stolzmann à Alausi le 25 avril. Iris brun foncé.

63. Zonotrichia pileata (Bodd.).

Un mâle pris par Stolzmann à Cechce le 1 mai.

*64. Chrysomitris capitalis, Cab.

Deux mâles de Cayandeled et de Cechce pris par Stolzmann en mars et mai.

L'oiseau de Cechce a les mêmes dimensions que les péruviens, mais l'autre exemplaire est plus petit, l'aile n'a que 61 mill. de longueur. L'viseau de Cechce a une particularité curieuse en ce qu'il a le milieu de la gorge jaune.

*65. Sycalis luteola (Sparrm.).

Deux mâles recueillis par Stolzmann à Cechce dans les premiers jours de mai. Iris brun foncé.

\section{ICTERID瓜.}

*66. Ostinops atrocastaneus, Cab. J. f. O. 1873, p. 309 (décrit de l'Ecuadeur).

Trois mâles recueillis à Pedregal en février. Iris brun foncé.

\section{Corvide.}

*67. Cyanocitta turcosa, Bp.

Deux oiseaux recueillis par Stolzmann à La Union (8700 pieds) en juin. Iris brun très foncé.

\section{Tyrannida.}

*68. Ochthodieta fumigatus (Boiss.).

Un mâle recueilli par Siemiradzki à La Union, en juin.

Identique aux oiseaux de Bogota (Musée Berlepsch), il a seulement le bec un peu moins fort.

[14] 
*69. Оснтнаса FUMicolor, Scl.

Deux mâles de Cechce recueillis par Siemiradzki en avril et mai. lris brun.

*70. Осhthøeca Lessoni, Scl.

Quatre mâles et trois femelles recueillis à Cechce, à La Union (8700 pieds), Tribulpata (7000 pieds) et à Chaguarpata, depuis le mois de mars jusqu'en juin. Un oiseau de Bogota (Musée Berlepsch) n'en diffère que par le brun du dos plus roussâtre, et le roux de la poitrine et de la bande transalaire plus pâle. Un oiseau de Quito paraît être intermédiaire. Il paraît donc que ce ne sont que des différences individuelles.

*71. Ochtheca Gratiosa, Scl., P.Z.S. 1862, p. 113 (décrite de l'Ecuadeur).

Deux mâles et une femelle de Chaguarpata, recueillis en janvier, février et mars.

Identiques à l'oiseau du Pérou septentrional et n'en sont distincts que par le brun du dos plus foncé, le jaune du front et du sourcil un peu plus vif; la bande rousse transalaire également distincte.

*72. Mecocerculus stictopterus (Scl.).

Une paire recueillie par Stolzmann à Cechce en avril.

Distincts des oiseaux péruviens par la couleur du dos d'un olive brunâtre obscur au lieu d'olive verdâtre.

73. Mecocerculus calopterus (Scl.).

Formicivora caloptera, Scl. P. Z. S. 1859, p. 142 (décrite de Pallatanga).

Serpophaga leucura, Lawr. Ibis, 1875, p. 384, pl. ix. f. 2 (décrite de l'Ecuadeur).

Deux femelles de Cayandeled recueillies le 18 et le 20 janvier. Iris brun foncé.

*74. Mecocerculus Pacrlocercus (Scl. et Salv.).

Une femelle recueillie par Stolzmann à Chaguarpata le 24 février. Iris brun foncé.

Exceptée une taille beaucoup moins forte, l'oiseau ressemble en tout aux péruviens.

*75. Muscisaxicola maculirostris (d'Orb. et Lafr.).

Deux femelles recueillies par Stolzmann à Yocon (8600 pieds) le 17 mai. Iris brun foncé.

*76. Pacilotriccus ${ }^{1}$ Rufigenis (Scl. et Salv.).

Todirostrum rufigene, Scl. et Salv. P. Z. S. 1877, p. 522 (décrit de Mongi).

Deux mâles de Cayandeled recueillis en janvier, février et mars. Iris rouge cerise très foncé.

1 Pøcilotriccus, g. nov., Berlepsch.

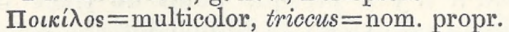

Species:-1 P. ruficeps (Kaup); 2 P. rufigenis (Scl. et Salv.). 
77. Lophotriccus squamicristatus (Berl.).

Deux mâles et une femelle de Pedregal et de Surupata, pris en janvier, février et mars.

*78. Anaretes parulus aquatorialis (Lafr.).

Deux mâles recueillis par Siemiradzki à Cechce le 29 avril et le 4 mai. Iris blanchâtre.

Il paraît que les oiseaux de l'Ecuadeur, identiques à ceux du Pérou septentrional, se distinguent des oiseaux du Chili par les plumes noires allongées de la huppe moins longues, le front et la nuque moins variés de blanchâtre, gorge et la poitrine marquées de taches noirâtres beaucoup plus larges, le dos d'un brun moins roussâtre (plus olivâtre), bec plus court et un peu plus élargi à la base.

*79. Mionectes striaticollis (d'Orb. et Lafr.).

Deux femelles de Surupata et de Chaguarpata recueillis en février.

80. Tyranniscus chrysops (Scl.).

Une paire et un jeune de Cayandeled recueillis en janvier et février.

*81. Tyranniscus uropygialis (Lawr.), Ann. Lyc. N. H. New York, 1869, p. 266.

Deux mâles et une femelle de Cechce et de Cerro Margarita, recueillis par Stolzmann en avril. Iris brun foncé. Selon toutes les donnéés il nous paraît que c'est un Tyranniscus et non Mecocerculus.

82. Ornithion sclateri, Berl. et Tacz.

Une femelle de Cayandeled prise par Stolzmann le 15 janvier.

*83. Elainea griseigularis, Scl., P.Z. S. 1858, p. 554, pl. cxlvi. f. 1.

Deux mâles et deux femelles pris par Stolzmann à Cechce et à Bugnac (5400 pieds) en mai.

Ces oiseaux se distinguent de la E. modesta, Tsch. (E. albiceps d’Orb.), du Pérou, par le blanc beaucoup moins développé sur la huppe interne, sans y former les cornes latérales foncées lorsque l'oiseau. hérisse les plumes; les deux bandes transalaires beaucoup plus régulières et mieux prononcées; pli de l'aile plus jaune; les flancs de l'abdomen lavés plus fortement de jaune; la couleur du dos plus foncée et tirant sur l'olivâtre. La taille est la même. Les oiseaux péruviens avec lesquels nos oiseaux ont été comparés ont été recueillis depuis novembre jusqu'en mars.

*84. Myiodynastes chrysocephalus, Tsch,

Un mâle pris par Stolzmann à Surupata le 19 janvier. Iris bru? foncé. Cet oiseau est d'une taille beaucoup moins forte que le mâle adulte du Pérou central, et s'en distingue par beaucoup de détails de la coloration, et principalement il a le devant du front fortement strié de blanc, tandis qu'il n'y a presque point de trace de cette couleur au front de l'oiseau péruvien; la huppe interne est orangée [16] 
au milieu, tandis qu'elle est toute limonacée dans l'oiseau péruvien ; le cendré de la nuque et du cou postérieur moins pur et lavé en grande partie d'olive; l'olive du dos plus foncé et tirant au brunâtre; les suscaudales entourées d'une bordure rousse; en dessous le bas de la gorge plus fortement coloré d'ocreux; les flammèches foncées pectorales beaucoup plus prononcées; le jaune du reste beaucoup plus intense; dans les ailes les tectrices et les remiges bordées plus fortement de roux à l'extérieur; la bordure interne des remiges est d'un ocreux roussâtre, tandis qu'elle est jaune à peine lavée de fauve chez l'oiseau péruvien; toutes les rectrices bordées finement à l'extérieur de roux, et plus largement au bord interne. Le bec est un peu plus élargi et moins atténué à l'extrémité.

La femelle du Pérou septentrional (Huambo) s'accorde en général avec l'oiseau du Pérou central, mais sous certains rapports elle est comme intermédiaire entre l'oiseau du Pérou central et celui de l'Ecuadeur, elle a beaucoup plus d'ocreux sur la gorge et le devant du cou, toutes les tectrices bordées des deux côtés de roux, mais beaucoup moins que chez l'oiseau de l'Ecuadeur.

ơ du Pérou central. Long. de l'aile 114, queue 97, bec 29, tarse 18 millim.

우 du Pérou septentrional. Long. de l'aile 110, queue 92, bec 29, tarse 18 millim.

ot de l'Ecuadeur occid. Long. de l'aile 97, queue 83, bec 29, tarse 17 millim.

*85. Mүiobius flavicans, Scl., P. Z. S. 1860, p. 464.

Six mâles et sept femelles de Cayandeled, Surupata (3800 pieds), Pedregal (2800 pieds), pris en janvier, février et mars.

86. Myiobius crypterythrus, Scl.

Un mâle et deux femelles de Cayandeled du 12 mars et du 27 février.

87. Myiobius stellatus, Cab.

Une paire de Cayandeled et de Pedregal pris par Stolzmann en janvier et en février.

88. Contopus ardesiacus (Lafr.).

Quatre mâles adultes, un jeune mâle et deux femelles de Cayandeled et de Bugnac, recueillis en janvier et février.

Identiques aux oiseaux du Pérou central et septentrional et de Venezuela (Musée Berlejsch); ce dernier a cependant les ailes un peu plus longues. Les oiseaux de l'Ecuadeur ont le milieu du bas ventre en général plus pâle et lavé de fauve.

89. Contopus richardoni (Sw.).

Un mâle de Pedregal (2800 pieds) et un jeune en mue de Place ${ }^{r}$ (2800 pieds) pris en janvier et en mars. Iris brun foncé.

90. Myiarchus nigriceps, Scl.

Un mâle de Bugnae (6000 pieds) pris par Siemiradzki en juin. 
91. Tyrannus melancholicus, Vieill.

Un mâle adulte de Cayandeled pris par Siemiradzki le 22 janvier.

Cotingide.

*92. Pachyrhamphus versicolor (Hartl.).

Un mâle et deux femelles de Cayandeled recueillis en février et mars.

Ce mâle a la gorge et les côtés de la tête lavés plus fortement de jaune que les exemplaires de Bogota et d'Antioquia (Musée Berlepsch), il a aussi le dessous du corps plus lavé de jaunâtre et les raies noires très peu apparentes. Les femelles ont le jaune plus intense que les femelles péruviennes, à raies foncées beaucoup plus marquées, le roux beaucoup plus repandu sur les ailes. En outre les dimensions sont plus petites que dans les oiseaux de la Nouvelle Grénade et du Pérou. Il paraît donc qu'il faudra les distinguer comme une race locale.

*93. Pipreola jucunda, Scl., P. Z. S. 1860, p. 89, pl. clix. (décrit de Cachi-Llacta près de Nanegal).

Six mâles et une femelle de Cayandeled (4500 pieds) recueillis en janvier et février. Iris jaune d'orange.

*94. Heliochera rubrocristata (d’Orb. et Lafr.).

Deux femelles de Cechce, prises en avril et mai. Iris rouge de cerise.

95. Cephalopterus penduliger, Scl.

Une femelle d'El-Placer (2600 pieds) prise par Siemiradzki le 24 février.

Dépourvue en entier de l'appendice jugulaire si remarquable chez l'autre sexe.

\section{DENDROCOLAPTIDE.}

*96. Synallaxis frontalis, Pelz.

Cinq mâles, trois femelles et un jeune de Cayandeled, de Chaguarpata et de Cechce, recueillis en février, mars et avril.

Ces oiseaux sont intermédiaires entre le $S$. frontalis du Pérou central et le $S$. fruticicola du Pérou septentrional. Ils sont tous aussi largement blancs au milieu des parties inférieures du corps comme cette dernière espèce; la bande sourcilière est d'un gris cendré plus clair que la région auriculaire; deux femelles ont même la partie postoculaire de cette bande colorée de roussâtre, mais moins fortement que chez les $S$. fruticicola.

97. Synallaxis pudica, Scl.

Un mâle recueilli par Siemiradzki à Cayandeled (4000 pieds) le 19 février. Iris brun rougeâtre.

98. Synallaxis erythrops, Scl.

Trois mâles et quatre femelles de Cayandeled et de Pedregal, recueillis en janvier, février et mars. Iris brun rongeâtre.

[18] 
*99. Synallaxis wyatti, Scl. et Salv., P. Z. S. 1870, p. 841.

Trois mâles et deux femelles de Cechce (10,400-11,000 pieds) recueillis en mai et en juin.

Ces oiseaux ne diffèrent d'un exemplaire de la Nouvelle Grénade (Santa Martha) du Musée de Berlepsch que par le bec moins long et plus foncé, l'aile plus courte, les taches noires des parties supérieures du corps en général plus grosses et moins largement bordées d'une nuance grise et un peu différente; le dessous du corps plus ocreux à stries ocreuses sur le derant du cou et la poitrine plus nettement prononcées sur un fond tirant au grisâtre; dans la queue nos oiseaux ont sur la quatrième rectrice une grosse ligne rousse beaucoup plus marquée sur la barbe interne près de la baguette. Quant à la tache gulaire la couleur n'est pas constante; un mâle l'a d'un roux ferrugineux assez intense parsemé finement de noir par les extrémités de ces plumes, en général beaucoup plus petite que dans les autres individus; le deuxième mâle l'a d'un ocreux roussâtre beaucoup plus grande que le précédent, également parsemée de noir ; le troisième mâle et la femelle l'ont d'un jaunâtre soyeux aussi pâle que celui de l'oiseau de la Nouvelle Grénade sans rien de noir au bout des plumes et sans bordure ocreuse en bas de cette tache, bien prononcée chez l'oiseau cité plus haut.

Un autre exemplaire provenant également de Santa Martha, de la collection de M. Sclater, présente les mêmes caractères différentiels dans la coloration, étant également en plumage frais, d'où vient que toutes les taches noires sont plus fines, et les bordures plus larges, mais il a le bec aussi court que tous nos oiseaux, l'aile au contraire un peu moins longue; le roussâtre de la tache gulaire intermédiaire entre celui des individus les plus pâles et celui qui l'a d'un ocreux roussâtre. Dimensions des oiseaux de l'Ecuadeur :-

ơ Longr. de l'aile 66, queue 68-70, bec 23, tarse $25 \mathrm{~mm}$.

ㅇ $, \quad 65, \quad, 68, \quad 21, \quad, 25$,

*100. Pseudocolaptes boissonneauti (Lafr.).

Une femelle de La Union ( 8700 pieds) pris par Stolzmann en juin.

*10l. Automolus holostictus striatidorsus, Stolzm. MS.

A. holosticto valde affinis, sed corpore subtus rufescentiore, minus olivaceo; alis extus rufescentioribus; rectricibus angustioribus; tectricibus superioribus et inferioribus caude saturatius brunneocastaneis; marginibus plumarum dorsi minus nigrescentibus et striis pallidis minus clare definitis; rostro, ut videtur, basi altiore vix distinguendus.

ơ . Long. de l'aile 86 , queue 92 , bec (culmen) 24 , tarse $26 \mathrm{~mm}$.

․ " " $85 \frac{1}{2}, " 84, \quad, 24, \quad, 26$,

Très voisin de l'A. holostictus, Scl. et Salv., d'Antioquia (Musée Berlepsch), mais différent dans les détails indiqués dans notre diagnose. M. Sclater, qui a examiné les oiseaux de Siemiradzki, les croit identiques à son $A$. holostictus. 
Deux mâles et une femelle de Cayandeled et de Chaguarpata recueillis en février et janvier. Iris brun foncé.

*102. Automolus ignobilis, Scl. et Salv. P. Z. S. 1879, p. 522 (décrit d'Antioquia).

Un mâle et trois femelles de Cayandeled (4100 pieds) recueillis en février et mars. Iris brun foncé.

M. Sclater, qui a comparé un exemplaire de l'Ecuadeur du Musée Berlepsch, identique aux oiseaux de Cayendeled, avec son type, n'a trouvé aucune différence.

103. Anabazenops temporalis (Scl.).

Une jeune femelle de Pedregal recueillie par Siemiradzki le 29 janvier. Iris brun.

*104. Anabazenops subalaris (Scl.), P. Z. S. 1859, p. 141 (décrit de Pallatanga).

Huit mâles et deux femelles de Cayandeled, de Pedregal (2800 pieds), de Surupata et de Chaguarpata (5700 pieds), recueillis en janvier, février et mars. Iris brun foncé.

*105. Margarornis perlata (Less.).

M. squamigera, auctt., nec d'Orb. et Lafr. (voyez 'Ibis,' 1874, p. 323).

Un mâle adulte de Chaguarpata recueilli par Siemiradzki le 24 janvier. Iris brun.

Le cannelle ou chatain du dessus du corps est plus intense, la gorge et les taches du dessous plus lavés de jaune que chez les oiseaux de Bogota. Il serait peut-être utile de séparer les oiseaux de l'Ecuadeur occid. comme une race locale.

106. Margarornis brunnescens, Scl.

Une paire et un oiseau sans indication de sexe de Cayandeled recueillis en janvier.

Identiques aux oiseaux de Chimbo, et présentent les mêrnes différences des oiseaux de la Nouvelle Grénade. Les oiseaux péruviens (Huambo) ont la queue plus longue que ceux de l'Ecuadeur, et les taches du dessous en général plus grosses.

107. Glyphorhynchus cuneatus castelnaudi (DesMurs).

Trois mâles et deux femelles de Cayandeled et de Pedregal (2600 pieds) recueillis en janvier et février. Iris brun foncé.

Identiques aux oiseaux de Chimbo. Ils diffèrent des oiseaux de Yurimaguas, recueillis également en janvier et février, par le bec plus court et plus large au bout, la nuance du dessous plus foncée, le roux de la gorge plus pâle et le cou antérieur et la poitrine plus fortement tachetés. Ces oiseaux de l'Ecuadeur ont en général la queue plus ou moins fortement usée à l'extrémité, tandis qu'elle est intacte dans les péruviens. Les oiseaux de Cayenne ont le bec aussi long et presque de la même forme que ceux de Yurimaguas, mais [20] 
s'en distinguent par le fond du dessous presque de la même nuance que celui des oiseaux de l'Ecuadeur et non olivâtre comme dans les oiseaux de Yurimaguas avec des stries claires prolongées le long du milieu du ventre jusqu'aux sous-caudales.

*108. Dendrocolaptes validus, Tsch.

Un mâle recueilli par Siemiradzki à Cayandeled le 19 janvier. Iris brun foncé.

Cet individu est jeune, et diffère beaucoup de l'oiseau de Bogota par la coloration en général plus obscure; le roux de la queue et des ailes plus foncé, les stries de la tête différentes; le bec plus large à la base et d'une couleur plus pâle.

Cet oiseau est d'une taille moins forte que ceux du Pérou centrul, à bec de la même forme et également élargi à la base; la couleur générale est plus foncée, surtout la queue présente une grande différence sous ce rapport; les stries fauves du dos sont distinctement plus larges, les raies brunes sur cette partie beaucoup moins déreloppées et reduites à des taches courtes disposées sur les deux côtés de la strie médiane; les stries du sommet de la tête sont d'une autre forme, c'est-à-dire qu'elles sont presque également larges dans toute leur longueur, tandis qu'elles sont très fines chez l'oiseau péruvien et fort élargies à l'extrémité.

Long. de l'aile 130, queue 115 , bec 45 , tarse $29 \mathrm{~mm}$. (ois. de Cayandeled).

Long. de l'aile 140, q'ieue 140, bec 45, tarse $28 \mathrm{~mm}$. (ois. de Monterico).

Les oiseaux du Pérou et de l'Ecuadeur ont été tués presque à la même époque de l'année, les premiers le 19 décembre, le dernier 19 janvier. Celui de l'Ecuadeur a l'extrémité des rectrices médianes fort usée.

L'oiseau d'Antioquia recueilli p r Salmon présente une différence beaucoup plus importante. Il a le bec beaucoup plus court et moins élargi; les raies du dos presque effacées, les raies du dessous plus fines.

Long. de l'aile 134, queue 122, bec $40 \mathrm{~mm}$.

*109. Picolaptes lacrymiger warszewiezi (Cab. et Hein.).

Quatre mâles de Cayandeled recueillis en janvier. Iris brun foncé.

Ces oiseaux de l'Ecuadeur ressemblent à ceux du Pérou et ne s'en distinguent que par la couleur des parties supérieures du corps plus rousse, sans rien d'olive qui se manifeste plus ou moins chez les oiseaux péruviens.

\section{Formicaritda.}

*110. Thamnophilus unicolor (Scl.).

Dysithamnus unicolor, Scl. P. Z. S. 1859, p. 141 (décrit de Pallatanga).

Quatre mâles adultes et deux femelles de Cayandeled et de Surupata recueillis en janvier, février et mars. Iris brun foncé.

Proc. Zool. Soc.-1884, No. XXI. 
L'oiseau typique de la collection de M. Sclater, examiné par Berlepsch, est identique en tout à nos oiseaux. M. Sclater est aussi actuellement d'avis que c'est un Thamnophilus et non Dysithamnus.

111. Dysithamnus semicinereus, Scl.

Un mâle moins adulte que celui de Chimbo recueilli par Stolzmann le 23 janvier. Iris brun foncé.

112. Myrmotherula menetriesi (d'Orb.).

Un mâle adulte pris par Stolzmann à Surupata le 27 janvier.

*113. Formicivora Caudata, Scl.

Cinq mâles, cinq femelles et deux jeunes de Cayandeled, Chaguarpata, et Surupata, recueillis en février et mars. Iris brun foncé.

114. Pyriglena picea, Cab.

Mâle adulte recueilli par Stolzmann à Cayandeled le 24 janvier. Iris rouge cramoisi.

Identique aux oiseaux péruviens.

*115. Grallaria ruficapilla, Lafr.

Deux mâles de Cayandeled et de Cechce, recueillis en janvier et mai. Iris brun foncé.

L'oiseau de Stolzmann a le roux de la tête moins foncé que les oiseaux d'Antioquia et de Medellin du Musée de Varsovie, et passant graduellement en olive du dos; le roux des côtés de la tête plus pâle; le blanc du dessous lavé de jaunâtre et non pur comme dans les oiseaux cités; les taches foncées des côtés de l'abdomen ont moins de noir et manquent en entier sur le milieu de la poitrine et de l'abdomen. L'oiseau est un peu moins fort, à bec distinctement plus long.

L'exemplaire de Siemiradzki et deux autres oiseaux du Musée de Berlepsch, recueillis dans l'Ecuadeur par Buckley, ont les couleurs du dessus (le roux de la tête et le brun olive du dos) plus obscurs qu'un oiseau de Bogota. Les premiers ont aussi les plumes du milieu de la poitrine moins bordées de brun et de roux, le fond du dessous d'un blanc plus pur. Le dernier a aussi l'ongle du doigt postérieur plus court.

*116. Grallaria regulus, Scl. et Salv.

Un mâle de Pedregal (2600 pieds) recueilli par Stolzmann le 27 février. Iris brun foncé.

La femelle du Pérou septentrional (Tambillo) se distingue de notre oiseau par la présence du blanc sur le collier, de grosses taches noires sur le milieu de la poitrine et des petites sur le wilieu de l'abdomen, le milieu de l'abdomen d'un roux beaucoup plus pâle, le fond du dos plus brunâtre, rnne tail:e moins forte.

*117. Grallaria monticola, Lafr.

Huit mâles, deux femelles, et un jeune en premier plumage de [22] 
Cechce jusqu'à 10,000 pieds, recueillis en avril et mai. Iris brun très foncé.

Identiques à l'oiseau de Quito au Musée Berlepsch.

*118. Grallaria rufula, Lafr.

Une paire de Cechce (10,000 pieds), recueillie par Stolzmann le 10 mai. Iris brun très foncé.

\section{Pteroptochida.}

*119. Scytalopus Magellanicus (Lath.).

Trois mâles adultes et une femelle de Chaguarpata (5700 pieds) et de Cechce. Iris brun foncé.

Identiques aux oiseaux de Cutervo.

\section{Trochilid A.}

*120. Phalemornis syrmatophorus, Gould.

Un mâle de Cayandeled, recueilli par Stolzmann le 20 janvier.

*121. Lafresnaya gayi (Bourc. et Muls.).

Deux mâles et deux femelles de Cayandeled et de Chaguarpata, recueillis en janvier, arril et mai.

*122. Petasophora anais (Less.).

Trois mâles adultes de Cechce et d'Alausi (7650 pieds), recueillis en avril et mai.

*123. Petasophora cyanotis (Bourc.).

Un mâle et deux femelles de Cayandeled et de Cechce, receuillis par Stolzmann en mars et avril.

*124. Pheolema equatorialis, Gould.

Huit mâles et trois femelles recueillis à Cayandeled en janvier, février, avril et mai.

Les femelles manquent de prase jugulaire, elles ont la gorge blanchâtre maculée de vert doré; la queue plus courte que celle du mâle.

*125. Eriocnemis luciani (Bourc.).

Un mâle et quatre femelles de Cechce, recueillis en avril et en mai.

*126. Heliotrypha viola (Gould).

Mâle et femelle de Cechce et de La Union (5700 pieds), recueillis en mai et juin.

*127. Diphlogena hesperus, Gould.

Treize mâles adultes, une femelle et trois jeunes mâles de Cechce et de Alpachaca (10,500 pieds), recueillis en avril et en mai.

․ La femelle se distingue du mâle d'une manière analogue à celle des deux autres espèces; les plumes du sommet de la tête sont 
courtes, moins polies et forment une surface comme squamuleuse d'une couleur semblable à celle du mâle, mais distinctement moins brillante, et presque uniforme depuis le front jusqu'à la nuque, c'est-à-dire qu'elle est plus rouge sur cette dernière que chez le mâle; la raie médiane bleue est plus claire et non saphirée, presque aussi large que celle du mâle et ne dépassant pas l'angle postérieur de l'œil, il lui manque donc la partie élargie sur le cervix propre à l'autre sexe; dans les autres directions de la lumière ce bleu passe en violet. Le vert des parties inférieures du corps est distinctement , lus clair que chez le mâle, mais également étincellant, même plus fortement sur l'abdomen; la base de toutes ces plumes est plus claire que chez le mâle, et en conséquence tout le dessous paraît être plus distinctement squamuleux; elle n'a aucune trace de la gemme jugulaire; le bronzé du dos moins uniforme, passant au vert sur les côtés. Le reste est comme chez le mâle. Les mâles non adultes se distinguent par le sommet de la tête couvert en entier de plumes aussi courtes que celles de la femelle d'un bronzé cuivreux obscur, à éclat métallique faible non étincellant, ou faiblement devant les yeux dans des rares directions de la lumière; le reste est comme chez les adultes; les uns ont la gemme jugulaire violette aussi bien développée que chez les adultes; les autres n'en ont point.

Il y a aussi un mâle à sommet de la tête paré d'une manière la plus brillante et la plus complète et sans gemme jugulaire, qui n'est répresentée que par une plume unique verte en partie et violette sur le reste.

*128. Panoplites mathewsi (Bourc.).

Un mâle et deux femelles de Cayandeled, recueillis en février et mars.

*129. Docimastes ensiferus schliephacker (Heine).

Deux mâles adultes et une femelle de Cechce, recueillis par Stolzmann en avril et mai.

*130. Bourcieria fulgidigula, Gould.

Six mâles et six femelles de Cayandeled et de Tribulpata (7000 pieds), recueillis en janvier, février et mars.

\section{Heliodoxa jamesoni (Bourc.).}

Un mâle de Placer (2800 pieds), recueillis par Stolzmann le 23 janvier.

132. Lampropygia wilsoni (Del. et Bourc.).

Deux paires de Cayarıdeled et de Pedregal, recueillis en janvier et février.

*133. Agleactis cupreipennis (Bourc. et Muls.).

Mâle et deux femelles, recueillis à Cerro Margarita et à Cechce en avril. 
*134. Metallura tyrianthina (Bourc.).

Quatre mâles et deux femelles de Chaguarpata (7000 pieds) et de Cechce, recueillis en janvier, février et avril.

Ces oiseaux s'accordent en tout à ceux de Bogota, ils ont seulement le dessous du corps un peu plus lavé de roussâtre. Les oiseaux de Quito sont plus grands, surtout le bec, et ont la queue plus dorée ; c'est le $M$. tyrianthina quitensis (Gould).

*135. Ramphomicron microrhynchum (Boiss.).

Un jeune mâle commençant à prendre l'habit d'adulte, recueilli par Stolzmann à Cechce le 6 mai.

*136. Adelomyia melanogenys maculata (Gould).

Un mâle et trois femelles de Cayandeled et un jeune mâle de Chaguarpata ( 5800 pieds), recueiliis en janvier, février ot mars.

En général ils ressemblent aux oiseaux de Bogota, mais ils ont la moitié basale de la barbe interne des rectrices latérales plus longuement blanc roussâtre clair et cette couleur mieux tranchée de la partie foncée. Il paraît aussi que dans cette race les taches gulaires sont plus grandes et plus prononcées, les flancs moins roussâtres, et le bec un peu plus long. Ils s'accordent aussi dans lès mêmes détails avec les oiseaux péruviers.

*137. Schistes personatus, Gould.

Quatre mâles de Pedregal et de Cayandeled, recueillis par Stolzmann en janvier et février.

\section{Cynanthus cyanurus celestis (Gould).}

Sept mâles adultes, deux jeunes mâles et cinq femelles de Cayandeled, recueillis en janvier et février.

*139. Lesbia amaryllis (Bourc.).

Cinq mâles et deux femelles de Cechce et d'Alausi, recueillis en avril et mai.

Identiques aux oiseaux de Quito (Musée Berlepsch), si ce n'est qu'ils ont les tectrices supérieures de la queue lavées de cuivreux doré au lieu d'améthyste violet, le vert du dos plus pur, moins doré et le bec un peu plus long.

Un mâle de Bogota (Musée Berlepsch) a la queue beaucoup moins longue que tous les oiseaux de l'Ecuadeur, mais en outre il ressemble en tout aux oiseaux de Quito. Si cette différence dans la queue serait constante on pourrait séparer la $L$. amaryllis de l'Ecuadeur comme une race locale.

*140. Lesbia gouldi gracilis (Gould).

Un mâle et trois fernelles de Cechce, recueillis en mai.

*141. Steganura melananthera (Jardine).

Sept mâles et six femelles recueillis à Cayandeled et à Pinyampunga et Surupata, en janvier, férrier et mars. 
Identiques aux oiseaux de Quito excepté qu'ils ont l'aile un peu plus longue et le vert de la gorge du mâle plus bleuâtre et plus terne, le front moins cuivreux. La forme et la couleur des palettes caudales varie beaucoup, dans les uns elles sont d'un beau bleu violâtre, dans les autres d'un bleu verdâtre.

*142. Acestrura mulsanti (Bourc.).

Jeune mâle commençant à prendre sa livrée d'adulte, pris par Stolzmann à Alausi le 24 avril.

143. Chatocercus bombus, Gould.

Deux paires d'oiseaux adultes de Cayandeled, prises en février et mars.

*144. Myrtis Fanny (Less.).

Deux mâles et une femelle pris par Stolzmann en mai à Bugnac (6400 pieds).

Cette femelle, incontestable selon Stolzmann, a sur un des côtés de la région jugulaire une plume squamiforme d'un bleu aussi brillant que celles du mâle.

145. Juliamyia feliciana (Less.).

Mâle adulte pris par Stolzmann à Bugnac le 28 mai.

146. Amazilia riefferi jucunda, Heine.

Six mâles et deux femelles de Cayandeled et de Pinampungu, recueillis en janvier, février et mars.

147. Chlorostilbon melanorhynchum, Gould.

Un jeune mâle pris par Stolzmann à Cayandeled le 15 janvier.

CAPRimuigide.

*148. Lurocalis rufiventris, Tacz. Ornith. du Pérou, i. p. 209.

Une paire recueillie par Siemiradzski à Cayandeled le 25 janvier.

Oiseaux identiques aux péruviens. Le mâle adulte se distingue de la femelle typique par les taches des parties supérieures du corps d'un roux moins foncé, la présence de quelques grosses taches blanches sur la nuque et le haut du cou postérieur couvertes pour la plupart par le brun noir de l'extrémité des plumes; les taches rousses du bas de la poitrine plus larges, transformées en raies transversales irrégulières; le roux du ventre un peu plus clair; les raies noires aux sous-caudales postérieures un peu plus larges; les extrémités des rectrices submédianes d'un roux ocreux au lieu de blanc; la remige externe sans aucunes taches sur les deux barbes; la seconde très faiblement tachetée. Longueur de l'aile $220 \mathrm{~mm}$.

149. Nyctidromus albicollis (Gm.).

Un mâle de Cayandeled, pris par Stolzmann le 12 janvier. Iris presque noir.

[26] 


\section{Picida.}

150. Campephilus sclateri (Malh.).

Un mâle de Cayandeled recueilli par Stolzmann en férrier.

*151. Campephilus pollens (Bp.).

Deux paires de Cayandeled et de Chaguarpata, recueillies en janvier et février. Iris jaune.

Ils diffèrent des oiseaux de Bogota (Musée Berlepsch) par les ailes et la quene plus courtes, le bec plus long. Eu coloration il n'y a rien de particulier.

*152. Chloronerpes fumigatus (d'Orb. et Lafr.).

Un mâle et deux femelles de Cayandeled et de El Placer (2800 pieds) pris en janvier et mars. Iris terre de Sienne brulée ou brun foncé.

*153. Chloronerpes malherbit, Scl.

Trois mâles et une femelle de Cechce (10,000 pieds) pris en avril, mai et juin. Iris brun foncé.

*154. Hypoxanthus rivolit brevirostris (Tacz.).

Une femelle de Cayandeled, recueillie par Siemiradzki en janvier. Iris brun rougeâtre.

Les oiseaux de l'Ecuadeur paraissent être intermédiaires entre lc vrai $H$. rivolii de Bogota et le vrai $H$. brevirostris péruvien. Ils ont le bec plus long que ceux du Pérou, mais ils s'en accordent par la dimension des ailes et de la queue et par la coloration. M. Dubois a dernièrement nommé l'oiseau de l'Ecuadeur $H$. aquatorialis, ce qui doit être rangé comme synonyme du H. brevirostris.

\section{Trogonide.}

*155. Trogon personatus propinquus (Cab. et Hein.), Mus. Hein. iv. p. 175.

Deux mâles de Cayandeled et de Chaguarpata (5700 pieds) et une femelle de Cayandeled, pris en janvier et février. Iris brun foncé chez le mâle, rouge cerise chex la femelle.

Cette forme du T'. personatus ne diffère de la forme typique de Bogota que par les dimensions un peu moins fortes, et plus sensiblement par le bec plus large et plus long. Le T. heliothrix du Pérou central a les mêmes proportions que le $T$. propinquus, et n'en diffère que par une nuance plus ou moins bleuâtre des parties supérieures du corps, de la poitrine et des rectrices médianes, le bec un peu plus comprimé et moins gros. Toutes ces races sont très proches entre elles.

156. Trogon virginalis, Cab. et Hein.

Deux mâles et une femelle de Pedregal (2800 pieds), recueillis en janvier et février. 
*157. Pharomacrus atjiceps heliactin (Cab. et Hein.), Mus. Hein. iv. p. 207.

Un mâle et une jeune femelle de Cayandeled (5000 pieds), pris en février. Iris du mâle brun foncé, gris de la femelle.

Comme ce mâle n'est pas complètement adulte et n'a qu'une partie de plumes fraiches sur la tête, on ne peut pas estimer la différence complète qu'il peut présenter des oiseaux péruviens ; il paraît cependant de ce qu'il y a que les plumes de la tête sont dorées, et non d'un doré cuivreux fort intense propre aux oiseaux du Pérou ; le doré des plumes du dos et des tectrices alaires est aussi plus faible. Les ailes dans cet oiseau de l'Ecuadeur sont moins longues de 8 millimètres; en revanche le bec est beaucoup plus fort.

*158. Pharomacrus antisiensis (d’Orb.).

Deux mâles et une femelle de Cayandeled recueillis en février. Iris rouge cerise.

Le mâle s'accorde en tout avec l'oiseau de Bogota du Musée Berlepsch, si ce n'est qu'il est un peu plus petit, et a le vert moins pur. Un oiseau de Sarayacu (Ecuador) recueilli par Buckley, et se trouvant au Musée Berlepsch, est encore plus petit et diffère essentiellement par un beau éclat cuivreux sur tout le vert du plumage, surtout sur la tête. Il possède aussi une bande sourcilière d'un cuivreux améthyste, manquant chez les autres oiseaux. Le dernier s'accorde le mieux avec la description de d'Orbigny, mais il faudra peut-être séparer l'oiseau de l'Ecuadeur occidental et de Bogota comme une race locale.

\section{Cuculide.}

159. Piaya cayana mesura (Cab. et Hein.)?

Un mâle de Surupata recueilli par Siemiradzki en janvier. Iris rouge cerise.

Parait être identique à l'oiseau de Bogota ; l'oiseau de Sta Lucia (Pérou septentrional) s'en accorde en tout.

\section{RAMPHASTIDE.}

*160. Andigena laminirostris, Gould.

Deux mâles et deux femelles de Chaguarpata (5800 pieds) et de Cayandeled, pris en janvier et février. Iris brun rougeâtre et brun autour de la pupille; pattes d'un vert olive. Les femelles ont le bec d'un tiers plus court que les mâles.

*161. Aulacorhamphus hematopygius, Gould.

Trois mâles et une femelle de Pedregal et de Cayandeled, pris en janvier et février. Iris terre de Sienne brulée.

Ces oiseaux diffèrent des deux exemplaires de Bogota (Musée Berlepsch) par le bec moins long et la mandibule supérieure enduite seulement de brun rougeâtre dans sa moitié latérale, tandis que dans les oiseaux de Bogota les côtés sont rouges jusqu'au bout. Il y a une strie bleuâtre au dessus de l'œil chez les individus de Bogota

[28] 
qui manque chez les oiseaux de l'Ecuadeur. Les derniers ont la poitrine plus fortement lavée de bleuâtre, sont généralement plus obscurs et moins forts dans toutes les dimensions.

\section{Psittacidas.}

*162. Pionus corallinus, Bp.

Deux mâles et une femelle recueillis par Stolzmann à Pedregal en février. Iris brun foncé.

*163. Pronus seniloides (Mass. et Souancé).

Une femelle tuée par Stolzmann à Cayendeled en février. Iris brun foncé.

\section{STRIgID}

*164. Bubo nigrescens, Berl.

B. virginiano ex America sept. et centr. proximus, sed differt colore supra subtusque nigrescentiore, minus rufescente, plumarum basibus ardesiacis (nec fulvis), tarsis fere unicoloribus sordide albis (nec fulvis fusco fasciatis), maculis albis apicalibus tectricum alurum superiorum fasciisque externis scapularium fere pure albis.

Tout le dessus du corps d'un brun noirâtre, varié de petites macules irrégulières d'un fauve pâle ou blanchâtre, disposées en bandes séparées (deux ou trois de chaque côté de la plume); la base des plumes en dessus et en dessous du corps est d'un ardoisé noirâtre (largement d'un fauve roussâtre chez le virginianus). Sommet de la tête presque uniforme sans maculature; les huppes également noirâtres avec une faible maculature ou une bordure blanchâtre au bord interne des plumes; les plumes basales du bec, la partie antérieure des tectrices auriculaires, le menton et une grande région jugulaire d'un blanc pur; les plumes des freins ont les baguettes noires, et quelques-unes au voisinage de l'œil sont noires en entier; la région oculaire et sourcilière mélangée de noirâtre et d'un blanc roussâtre; tectrices auriculaires antérieures terminées largement d'une bordure noire formant un croissant noir très distinct sur chacun des côtés de la tête; les tectrices postérieures mélangées de blanc et de noirâtre. Gorge bordée en dessous d'une bande étroite de plumes obscures avec une maculature fauve; les côtés du cou, le bord supérieur et les côtés de la poitrine ont la même coloration que le dos; sur la poitrine inférieure les raies blanches sont plus grosses et plus distinctes, formant des bandes régulières sur les plumes; ces bandes blanches deviennent sur l'abdomen encore plus larges, de sorte qu'on pourrait nommer les plumes blanches rayées régulièrement de bandes noirâtres (5-7 sur chaque plume, plus étroites que les intervalles blanchâtres, séparées du noirâtre par une ligne fauve); la région anale, les flancs internes et les sous-caudales variés d'une manière semblable au ventre, mais d'une teinte générale plus roussâtre. Les plumes des tibias d'un fauve blanchâtre avec une petite maculature obscure peu visible; les plumes du tarse d'un 
blanc sale uniforme, sans taches. Remiges et rectrices d'un brun noirâtre comme le dos, traversées de bandes d'un brun grisâtre mélangé de roussâtre et de blanchâtre, ondulé et moncheté de noirâtre. Sur la barbe interne des remiges et des rectrices, excepté les deux médianes, ces bandes sont plus larges, et d'une couleur fauve presque en entier mélangée de noirâtre. Les rectrices terminées largement à peu près sur 18-20 millimètres, d'un blanc ronssâtre presque uniforme, excepté les d’eux médianes, qui ont l'extrémité mélangée de blanc et de noirâtre; il y a 6 bandes claires sur les rectrices qui paraissent être d'un blanc roussâtre uniforme sur la page inférieure et y présentent la même largeur que les bandes noirâtres. Tectrices supérieures de la queue noirâtres barrées et ondulées irrégulièrement de blanc roussâtre. Les petites tectrices supérieures de l'avant bras sont d'un noirâtre peu varié de roussâtre; celles des remiges primaires et de l'aile batarde noirâtres avec une ou deux larges bandes d'un fauve clair sur la barbe interne; les moyennes et les grandes noirâtres mélangés de blanc roussâtre, les antérieures présentant une grosse tache d'un blanc presque pur sur l'extrémité de la barbe externe, mouchetée un peu de noirâtre en arrière; les scapulaires externes ont aussi quelques taches blanchâtres, mais variés plus de roussâtre en dehors; les sous-alaires antérieures barrées de noirâtre et de fauve, les postérieures blanches mélangées de noirâtre. Trois bandes claires sur la page inférieure de la première remige. Bec noirâtre, à extrémité pâle ; iris jaune.

Long. totale 460 , aile 350 , queue 185 , bec (culmen) $30 \frac{1}{2}$, tarse $80 \mathrm{~mm}$.

Une femelle de Cechce (10,000 pieds) prise par Siemiradzki en juillet.

Au premier coup d'œil on est tenté de prendre cet oiseau pour une variété nègre, comme on a souvent des exemples dans la classe des rapaces; mais comme M. Sharpe, dans le Catalogue des Striges (p. 23), parle d'un oiseau pareil, provenant aussi de l'Ecuadeur, du Musée Salvin et Godman, nous nous croyons être justifiés de l'opinion que cette curieuse coloration peut être constante et propre à une forme de Bubo habitant les montagnes de l'Ecuadeur occidental, et non encore reconnue.

*165. Micrastur guerilla, Cass.

Une femelle de Surupata prise par Siemiradzki le 24 janvier. Iris brun grisâtre; parties nues de la tête et pattes jaunes.

Long. de l'aile 172, queue 165, bec (culmen) $17 \frac{1}{4}$, tarse $58 \mathrm{~mm}$.

Le doigt externe sensiblement plus long que l'interne.

Cet oiseau diffère de deux oiseaux adultes du $M$. guerilla, prove. nants de Guatemala (Musée Berlepsch), par le plumage généralement plus obscur. Tête, dos et les ailes à l'extérieur sont d'un ardoisé brunâtre uniforme, tandis que chez les oiseaux de Guatemala le dos et la tête sont d'un ardoisé un peu plus clair, les ailes d'un brun chocolat un peu roussâtre. En dessous la différence est encore plus frappante, car les raies noirâtres sont plus larges et plus rapprochées entre elles, les blanchâtres plus étroites et fines sur la poitrine, ce [30] 
qui fait que tout le dessous du corps a une apparence beaucoup plus foncée. La gorge est d'un brun assez sombre (plombé clair chez le $M$. guerilla). Il n'y a que des traces faibles de deux bandes blanchâtres sur les rectrices médianes au lieu de trois bien prononcées de $\boldsymbol{M}$. guerilla. On ne voit pas de différence dans les dimensions, mais le bec de l'oiseau de Surupata est un peu plus long.

Cet oiseau de Surupata pourrait aussi appartenir au M. pelzelni, Ridgw. ('Ibis,' 1876), décrit du Pérou oriental, car il a la queue plus courte que l'aile et n'a que deux bandes peu distinctes sur les rectrices, ce qui constitue les caractères distinctifs indiqués par M. Ridgway entre le M. pelzelni et le guerilla. Quant à la longueur de lı queue, il faut remarquer que les deux oiseaux adultes de Guatemala (Musée Berlepsch) ont aussi la queue plus courte que l'aile, et qu'us troisième jeune de la même localité l'a de la même longueur que l'aile.

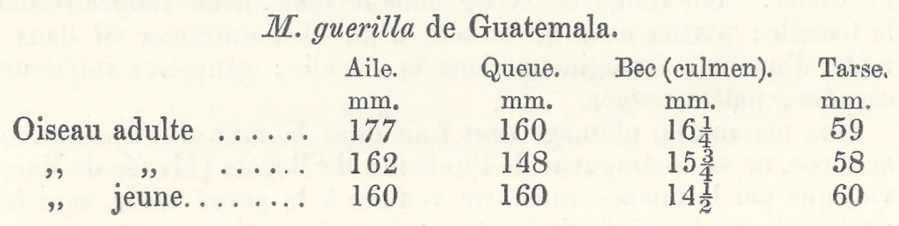

\section{Columbid}

*166. Columba albilinea, Gr.

Une femelle recueillie par Siemiradzki à Bugnac.

67. Zenaida maculata (Vieill.).

Deux mâles de Cechce recueillis en mai.

*168. Metriopelia melanoptera (Molina).

Un mâle recueilli par Siemiradzki à Cechce le 1 mai.

Coloration plus obscure et plus brunâtre que chez les oiseaux du Pérou méridional et de Chili au Musée Berlepsch.

*169. Leptoptila verreauxi, Bp.

Mâle adulte de Pedregal et un jeune mâle de Cayandeled, recueillis en février. Iris jaune orangé.

Différent un peu des oiseaux de l'Amérique centrale par les conleurs généralement plus obscures, et les sous-caudales bordées d'une nuance brunâtre, la couleur du dessous des ailes d'un roux plus foncé.

Des oiseaux du Pérou septentrional il diffère aussi par la couleuı des parties supérieures du corps et des ailes tirant un peu au brunâtre; tout le dessous du corps lavé plus fortement de rose; l'éclat rosé beaucoup plus fort sur la nuque et le cou postérieur, le rose commençant sur le vertex vis-à-vis de l'angle antérieur des yeux; couleur rosée et non roussâtre sur les côtés du cervix et de la nuque; 
le roux $d u$ dessous des ailes plus intense et tirant au rougeâtre; les sous-caudales bordées de brunâtre.

*170. Geotrygon bourcieri, Bp.

Un mâle recueilli par Stolzmann à La Union en janvier.

\section{Cracidar.}

*171. Penelope montagnit, Bp.

Une paire d'adultes recueillie par Siemiradzki à La Union (87009000 pieds) en juin et juillet.

\section{*172. Cham apetes goudoti (Less.).}

Quatre mâles et deux femelles de Cayandeled recueillis en janvier et février. Iris rouge de cerise dans le mâle, brun rouggâtre dans la femelle; parties nues de la tête d'un bleu outremer vif dans le mâle, d'un noir ferrugineux dans la femelle; paupières inférieures carnées; pattes rouges.

Nos oiseaux en plumage tout frais dont la mue n'est pas encore achevée, ne se distinguent de l'individu de Bogota (Musée de Varsovie) que par la nuance roussâtre réduite à la gorge seule, sans être prolongée sur les côtés de la tête et du cou postérieur comme cela a lieu chez l'oiseau cité ; le roux du dessous paraît être un peu plus rougeâtre, ne commençant pas aussi haut sur le bas de la région jugulaire. La troisième remige primaire est beaucoup moins attenuée dans ces oiseaux de l'Ecuadeur, ce qui ne se voit qu'au bout même.

\begin{tabular}{|c|c|c|c|c|c|}
\hline & Aile. & Queue. & Bec. & Tarse. & \\
\hline$\sigma^{*} \ldots$ & $\begin{array}{l}\mathrm{mm} . \\
240\end{array}$ & $\begin{array}{l}\mathrm{mm} . \\
240\end{array}$ & $\begin{array}{c}\mathrm{mm} . \\
38\end{array}$ & $\begin{array}{c}\mathrm{mm} . \\
62\end{array}$ & \\
\hline$\sigma^{\pi} \ldots$. & 250 & 220 & 35 & 65 & de Cayandeled. \\
\hline ㅇ...... & 230 & 230 & 35 & 65 & \\
\hline ? . . . & 240 & 237 & 38 & & $\begin{array}{l}\text { de Bogota. } \\
\text { de Govinda. Ecua- }\end{array}$ \\
\hline$\sigma^{\pi}$. & 263 & 280 & - & 62 & $\begin{array}{l}\text { dor (coll. Buck- } \\
\text { ley). }\end{array}$ \\
\hline
\end{tabular}

Ce dernier sera probablement identique au Ch. tschudii, Tacz., du Pérou septentrional.

*173. Crypturus transfasciatus, Scl. \& Salv. P. Z. S. 1878, p. 141, pl. xiii. (décrit de Sta Rosa, Ecuador).

Une paire de Guayaquil recueillie en août. Iris rougeâtre.

*174. Nothoprocta curvirostris, Scl. \& Salv. Nomencl. Av. Neotr. p. 163 (décrit de Calacali et Puellaro).

Une paire de Cechce (10,400 pieds) recueillie en avril et mai. Iris brun foncé. 


\section{RALLID E.}

*175. Rallus virginianus, L.

Mâle adulte de Yoyacsi tué par Stolzmann en juin à 9000 pieds d'altitude. Iris brun rougeâtre.

*176. Porzana carolina (L.).

Une paire recueillie par Stolzmann à Cayandeled en février.

\section{SCOLOPACIDA.}

*177. Gallinago nobilis, Scl.

Deux mâles et une femelle adultes et un jeune mâle recueillis à Yoyacsi (9000-9100 pieds) en juin. Iris presque noir.

Comparés avec deux oiseaux de Bogota (Musée de Varsovie) et on ne voit pas aucune différence.

Toutes les espèces non fournies dans l'envoi précédent sont marquées par un astérique, comme il y en a 127 de pareilles daus cette liste, le nombre d'espèces recueillis par nos voyageurs est de 341 .

\section{Notes Supplémentaires À notre Première Liste des Oiseaux de l'Ecuadeur occidental.}

1. Hylophilus minor, Stolzm. P. Z. S. 1883 , p. 542.

L'oiseau de la collection de M. Sclater, recueilli par Fraser à Bubahayo et nommé par M. Sclater Hylophilus sp.?, et H. aurantiifrons?, a été examiné par Berlepsch. C'est un jeune de notre H. minor.

2. Phøintcothraupis stolzmanni, nobis, P. Z.S. 1883, p. 546.

M. Salvin en examinant la femelle recueillie par Siemiradzki était de l'opinion qu'elle appartenait au Ch. olivacea (Cass.) d'Antioquia et du Rio Truando (voyez Biolog. Centr.-Am., Aves, p. 298).

Nous avons déjà expliqué qu'il n'y a pas de différence entre les deux sexes de l'oiseau recueilli par Stolzmann et Siemiradzki, tandis que tous les individus du Ch. olivacea connus, et que M. Salvin croit être mâles, ont la gorge, les freins, tour de l'œeil et une partie des sousalaires jaunes. On ne voit rien de pareil chez nos oiseavx des deux sexes, qui paraissent constituer une bonne espèce. pl. 1.

3. Chrysomitris siemiradzkit, nob. P. Z. S. 1883, p. 551 ,

M. Sclater reconnait nos oiseaux pour une excellente espèce, et dit qu'il ne possède dans sa collection qu'une femelle de Cuenca (Fraser). Les oiseaux de Pallatanga nommés dans la liste de M. Sclater $C h$. icterica appartiendront probablement au $C h$. capitalis, Cab.
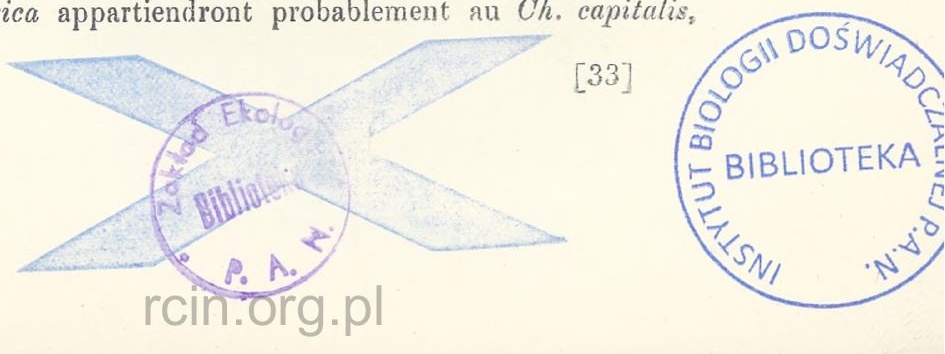
rcin.org.pl 


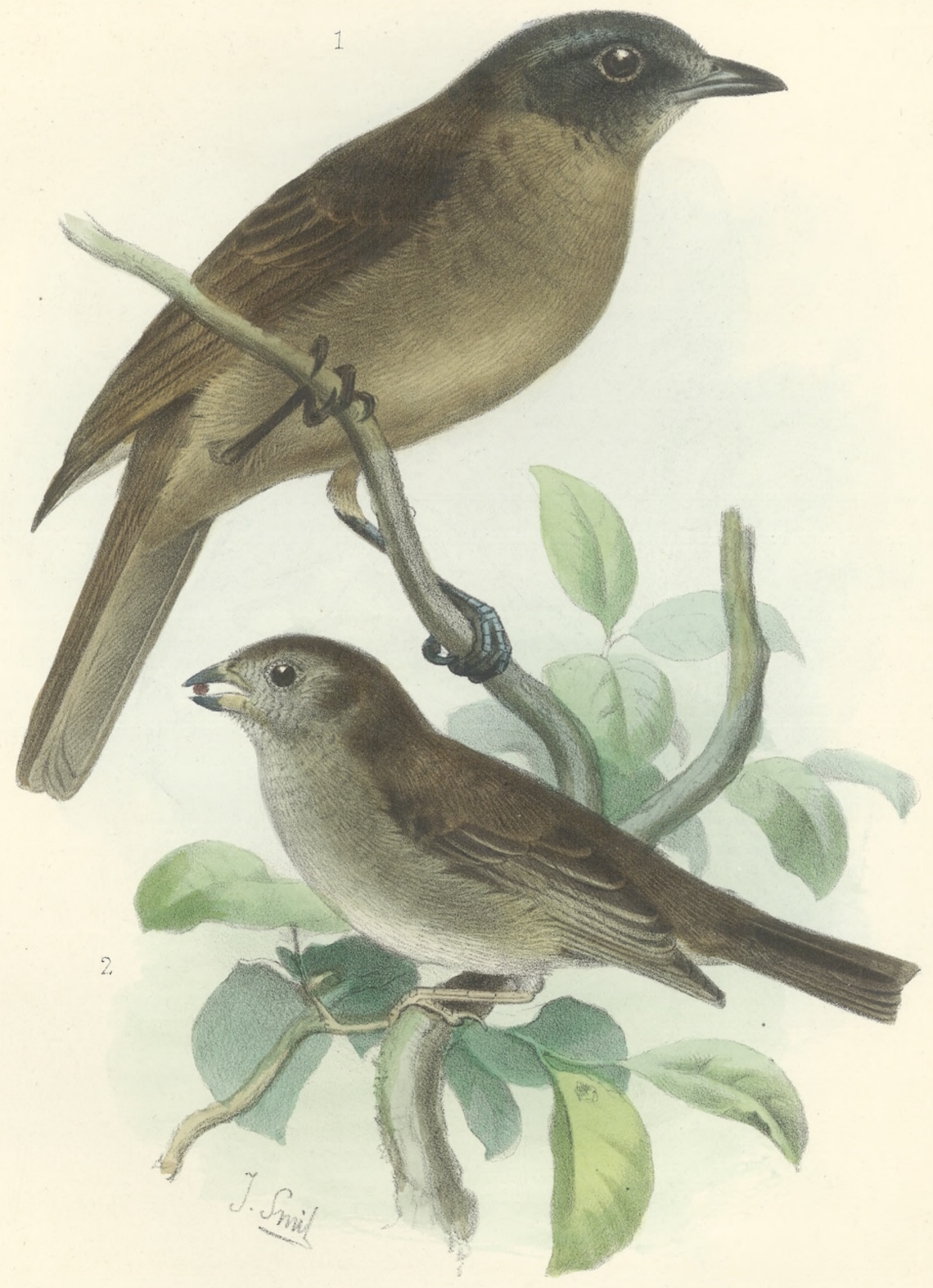


rcin.org.pl 
rcin.org.pl 
rcin.org.pl 